\title{
Effect of dexmedetomidine on early postoperative cognitive dysfunction and peri-operative inflammation in elderly patients undergoing laparoscopic cholecystectomy
}

\author{
YUHONG LI ${ }^{1,2}$, RUI HE ${ }^{1}$, SHUNFU CHEN ${ }^{1}$ and YULIAN QU ${ }^{3}$ \\ ${ }^{1}$ Department of Anesthesiology and ${ }^{2}$ Medical Research Center, Shaoxing People's Hospital, Shaoxing, Zhejiang 312000; \\ ${ }^{3}$ Department of Anesthesiology, The Second People's Hospital of Wenling City, Wenling, Zhejiang 317502, P.R. China
}

Received September 8, 2014; Accepted July 17, 2015

DOI: 10.3892/etm.2015.2726

\begin{abstract}
The use of intravenous dexmedetomidine during surgery has been shown to suppress inflammatory cytokines peri-operatively. It has also been demonstrated that dexmedetomidine may benefit cognitive function in elderly patients following surgery; however, it is not clear whether dexmedetomidine reduces postoperative cognitive dysfunction (POCD) via the suppression of inflammatory cytokines. The aim of the present study was to investigate the effects of dexmedetomidine on early POCD and inflammatory cytokines in elderly patients undergoing laparoscopic cholecystectomy (LC). The study comprised 120 elderly patients undergoing selective LC, who were randomly allocated to receive either dexmedetomidine intravenously (DEX group, $n=60$ ) or the same volume of normal saline (control group, $\mathrm{n}=60$ ). Cognitive function was assessed by Mini-Mental State Examination (MMSE) scores 1 day prior to surgery, $6 \mathrm{~h}$ following surgery and postoperatively on days 1 and 2 . Interleukin (IL)-1 $\beta$, IL-6 and C-reactive protein (CRP) levels were also measured at these time-points. On the basis of whether the patients had POCD on the first day after surgery, patients were divided into a POCD group and a non-POCD group. Blood cytokine levels were compared between the patients with and without POCD. A total of 100 patients completed both pre- and postoperative MMSE tests. At 1 day following surgery, POCD occurred in 10/50 (20\%) patients in the DEX group and in 21/50 (42\%) patients in the control group $(\mathrm{P}=0.017)$. At $6 \mathrm{~h}$ following surgery, IL-1 $\beta$, IL- 6 and CRP levels showed significant increases $(\mathrm{P}<0.01)$ compared with the baseline levels in the two groups. Furthermore, in the control group, CRP levels showed a significant increase on day $1(\mathrm{P}<0.001)$ and day $2(\mathrm{P}=0.017)$
\end{abstract}

Correspondence to: Dr Yulian Qu, Department of Anesthesiology, The Second People's Hospital of Wenling City, 68 Jiangnan Road, Wenling, Zhejiang 317502, P.R. China

E-mail: 1152305176@qq.com

Key words: dexmedetodine, postoperative cognition dysfunction, elderly patients, cytokines postoperatively. In the DEX group compared with the control group, IL-1 $\beta$, IL- 6 and CRP levels were markedly decreased at $6 \mathrm{~h}$ and 1 day after surgery $(\mathrm{P}<0.01)$. Concentrations of IL-1 $\beta$, IL-6 and CRP were significantly higher in patients who developed POCD on day 1 following surgery than in the patients who did not develop POCD $(\mathrm{P}<0.05)$. The findings of the current study support the hypothesis that dexmedetomidine administration during anesthesia decreases the incidence of early POCD, most likely by the mechanism of reduction of the inflammatory response level.

\section{Introduction}

Due to a rapid increase in the aging population, the amount of surgery that is now performed in elderly patients accounts for $25-30 \%$ of all surgical procedures (1). Postoperative cognitive dysfunction (POCD) is a common postoperative neurological complication in elderly patients with an incidence of 5-15\% (2). POCD is a subtle impairment of memory, concentration and information processing with clinical manifestations of delirium, anxiety, personality changes and impaired memory, which is associated with prolonged hospitalization, a reduced quality of life and an increase in morbidity and mortality (3-7). Previous studies have suggested that age, surgery itself and anesthesia may be the possible causes of POCD $(6,8)$; therefore, POCD has become a major concern for the anesthetist. Evidence has shown that an inflammatory increase, particularly the inflammatory response in the central nervous system, may serve as a predictive parameter for the occurrence of POCD (3,9-14). A meta-analysis has indicated that interleukin (IL)-6 may serve as a biomarker to provide guidance in the prevention and treatment of POCD (15).

Dexmedetomidine is a highly selective $\alpha-2$ adrenergic receptor agonist with a dose-dependent sedative hypnotic effect. A conventional treatment dose of dexmedetomidine has analgesic properties and inhibits sympathetic activity without causing respiratory depression (16-18). It has also been reported that dexmedetomidine has a potential role in preventing POCD due to its neuroprotective effects both in vitro and in vivo (19).

In the present study, it was hypothesized that dexmedetomidine reduces the incidence of POCD by decreasing the inflammatory response. In order to validate the hypothesis, 
the incidence of POCD was investigated and the inflammatory cytokines were analyzed during combined intravenous dexmedetomidine general anesthesia in elderly patients undergoing laparoscopic cholecystectomy (LC).

\section{Subjects and methods}

Ethics considerations. The protocol was approved by the Ethics Committee of Shaoxing People's Hospital (Shaoxing, China). The study was conducted in accordance with the guidelines of Good Clinical Practice and the principles expressed in the Declaration of Helsinki. Each subject gave his or her consent for participation after being informed of the study orally and in writing prior to the surgery.

Patient recruitment. Between March 2010 and July 2012, 120 patients were recruited to participate in the study. The patients were randomly assigned to either the experimental group (DEX group, $n=60$ ) or the normal saline group (control group, $n=60$ ) by a statistician. Inclusion criteria were: i) Patients with American Society of Anesthesiology (ASA) status of I-III; ii) aged $>60$ years old; and iii) scheduled for elective LC under intravenous general anesthesia. Exclusion criteria were: i) Patients were aged $<60$ or $>75$ years; ii) patients with accompanying medical conditions that may affect the level of consciousness, such as stroke, stupor or dementia, or patients with abnormalities in hepatic or renal function; iii) patients suffering from preoperative bradycardia [heart rate (HR) $<45 \mathrm{bpm}$ ] or hypotension [mean arterial blood pressure (MAP) $<60 \mathrm{mmHg}$; iv) patients who had recently received a sedative or opioid drug; and v) patients with a Mini Mental State Examination (MMSE) score $<24$.

Study design. This was a prospective, randomized, placebo-controlled, double-blind clinical trial. The patients recruited according to the inclusion criteria were distributed to one of the groups according to computer-generated random assignment. The personnel involved in the study, including statisticians, investigators, anesthetists, surgeons and the patients were blinded to the specific experimental scheme implementation.

Procedures. Patients were fasted overnight and no premedication was given. When the patients entered the operating theater, catheterization of the left radical artery and a large vein in the right forearm was performed. Anesthesia was induced with fentanyl $(2-3 \mu \mathrm{g} / \mathrm{kg})$, midazolam $(0.04-0.05 \mathrm{mg} / \mathrm{kg})$ and target-controlled infusion (TCI)-guided propofol targeting a plasma concentration of 3-4 $\mu \mathrm{g} / \mathrm{ml}$. Tracheal intubation was facilitated with $0.15 \mathrm{mg} / \mathrm{kg}$ cis-atracurium. The patients were mechanically ventilated and end-tidal $\mathrm{CO}_{2}$ was held constant between $35-40 \mathrm{mmHg}$. Following the induction of general anesthesia, patients in the DEX group were administered dexmedetomidine at a dose of $1 \mathrm{mg} / \mathrm{kg}$ over $10 \mathrm{~min}$, followed by a continuous infusion at a rate of $0.4 \mathrm{mg} / \mathrm{kg} / \mathrm{h}$ until the end of surgery. The control group received a placebo infusion of normal saline. Anesthesia was maintained with $1-2 \%$ end-tidal sevoflurane together with TCI of propofol (target plasma concentration, $2-3 \mu \mathrm{g} / \mathrm{ml}$ ), continuous infusion of remifentanil $(0.10-0.20 \mu \mathrm{g} / \mathrm{kg} / \mathrm{min})$ and cis-atracurium intermittently as required. Propofol and remifentanil were discontinued $5 \mathrm{~min}$ prior to the end of surgery, while the infusion of dexmedetomidine or normal saline was stopped at the end of surgery. The patients were transported to the post-anesthesia care unit and administered neostigmine $(0.03 \mathrm{mg} / \mathrm{kg})$ and atropine $(0.015 \mathrm{mg} / \mathrm{kg})$ to reverse neuromuscular blockade subsequent to swallowing reflex recovery. Tracheal extubation criteria were: i) Spontaneous breathing with tidal volume $>6 \mathrm{ml} / \mathrm{kg}$ and breathing rate $<30 \mathrm{bpm}$; ii) partial pressure of end-tidal carbon dioxide $\left(\mathrm{PETCO}_{2}\right)$ was maintained at $35-45 \mathrm{mmHg}$ with presence of a normal shape of capnographic curve (elance 7c, Spacelabs Medical Inc, USA); iii) pulse oximetry $\left(\mathrm{SpO}_{2}\right)$ was maintained at $>92 \%$ under air inspiration; and iv) recovery of consciousness and the ability to form a strong fist.

Measurements and blood samples. During the investigation, $\mathrm{HR}$, electrocardiography (ECG), MAP, $\mathrm{SpO}_{2}$ and $\mathrm{PETCO}_{2}$ were continuously monitored. The depth of anesthesia was monitored and recorded by a Bispectral Index ${ }^{\mathrm{TM}}(\mathrm{BIS})$ sensor (BIS monitor Model A-2000; Aspect Medical System, Norwood, MA, USA) applied to the forehead. The anesthesia was guided to reach a BIS value of 40-60. Body temperature was maintained at $\geq 35.5^{\circ} \mathrm{C}$. In the case of hypotension, which was defined as a reduction of systolic blood pressure by $>30 \%$ of the pre-anesthetic value, $<90 \mathrm{mmHg}$ ephedrine was administered, while $0.5 \mathrm{mg}$ atropine was given if the HR fell to $<50 \mathrm{bpm}$. The treatments were repeated if necessary. Cognitive function was evaluated with the MMSE immediately prior to the induction of anesthesia (T0), and at $6 \mathrm{~h}$ (T1), $24 \mathrm{~h}$ (T2) and $48 \mathrm{~h}$ (T3) postoperatively (20). Preoperative assessment, including disease duration, co-morbid diseases and medication at home was completed the day prior to surgery. Venous blood (10 $\mathrm{ml}$ at each time-point) was drawn to determine the concentrations of C-reactive protein (CRP), IL-1 $\beta$ and IL-6 as representative systemic inflammatory mediators at $\mathrm{T} 0, \mathrm{~T} 1, \mathrm{~T} 2$ and T3. Blood samples were drawn via an indwelling catheter inserted into the forearm vein and put into heparin anticoagulant tubes, transported to the hospital research laboratory within $30 \mathrm{~min}$, and then centrifuged at 2,000 $\mathrm{xg}$ for $10 \mathrm{~min}$ at $4^{\circ} \mathrm{C}$. Separated plasma samples were stored at $-80^{\circ} \mathrm{C}$ until assayed. Using commercially available ELISA kits, plasma levels of the following cytokines were measured using ELISA, 70-E-EK 101B2 Human IL-1 $\beta$ ELISA Kit, MultiSciences Biotech Co., Ltd, Hangzhou, China), IL-6 (using ELISA, 70-E-EK1062 Human IL-6 ELISA Kit, MultiSciences Biotech Co., Ltd, Hangzhou, China), CRP (using Immunoturbidimetry, CRP Kit, Meikang Biotech Co., Ltd, Ningbo, China ).

Outcomes. The main outcome of the study was cognitive function, which was defined according to the MMSE score. Compared with baseline, postoperative POCD was considered according to the criteria of MMSE score reductions of $\geq 1 \pm$ standard deviation, as described by Newman (21). Cognitive impairment was defined and graded according to the MMSE score: $<27$ indicates cognitive impairment; 21-27, mild cognitive impairment; 9-21, moderate cognitive impairment; and $<9$, severe cognitive impairment. On the basis of whether the patients had POCD in the first day subsequent to surgery, patients were divided into POCD and non-POCD groups, and a comparison was made between the outcomes of these two groups. 
Statistical analysis. Quantitative variables are presented as mean \pm standard deviation or median with interquartile range. Categorical variables were analyzed using $\chi^{2}$ or Fisher's exact tests. Continuous variables were tested with MannWhitney $\mathrm{U}$ tests or Student's t-tests depending on the distribution of the data. Repeat analysis of variance was used to compare the difference between the different times in the two groups. $\mathrm{P}<0.05$ was considered to indicate a statistically significant difference and adjusted for multiple comparisons when appropriate. The following software packages were used to perform analysis: Excel 2000 (Microsoft, Redmond, MA, USA) and SPSS version 10.0 (SPSS Inc., Chicago, IL, USA).

\section{Results}

Comparison between DEX and control groups. From March 2010 to July 2012, 120 elderly patients were recruited in accordance with the inclusion criteria. However, 20 patients were subsequently excluded: 7 patients declined to give consent; surgery was cancelled for 5 patients and surgery was abandoned for another 8 patients. The remaining 100 patients were included in randomization and data analysis (Fig. 1). No significant differences in pre-test variables such as demographic and surgical details were observed between the DEX and control groups (Table I).

Table II illustrates that the preoperative MMSE scores and the scores on day 2 after surgery were similar in the two groups $(\mathrm{P}>0.05)$. However, compared with the control group, the MMSE scores at $6 \mathrm{~h}$ and 1 day after surgery were significantly higher in the DEX group $(\mathrm{P}<0.001, \mathrm{P}=0.012$, respectively). Postoperatively 19 patients in the control group and 9 patients in the DEX group developed mild cognitive impairment $(\mathrm{P}=0.026)$. The number of patients with moderate cognitive impairment in the DEX and control groups was 1 and 2 patients, respectively, with no significant difference between the groups $(\mathrm{P}=0.558)$. None of the patients developed severe cognitive impairment (Table III).

Hemodynamic data. Dexmedetomidine administration resulted in significant reductions in HR and MAP at the end of peritoneal closure (T2, the definition is different from that in the rest of the paper, which is 1 day after surgery) $(\mathrm{P}=0.006$ for MAP; $\mathrm{P}=0.003$ for HR). There were no significant differences in MAP and HR observed between the DEX and control groups at the time-points of T0 (prior to induction of anesthesia) and T1 (10 min following infusion of study drug, this definition is different from that in the rest of the paper, which is $6 \mathrm{~h}$ after surgery; Fig. 2).

The baseline plasma values of the cytokines IL-1 $\beta$, IL-6 and CRP were comparable in the DEX and control groups; however, the concentrations of IL-1 $\beta$, IL- 6 and CRP were increased significantly $6 \mathrm{~h}$ after surgery and 1 day after surgery in the control group compared with those in the DEX group (Fig. 3). When compared with baseline values, the levels of IL-1 $\beta$, IL-6 and CRP were markedly increased at the 6-h time-point for patients in the DEX group, while they were increased at $6 \mathrm{~h}$ and 1 day following surgery for patients in the control group. Two days postoperatively, IL-1 $\beta$ and IL- 6 levels were not significantly different from baseline levels in the control group, while CRP levels remained marginally

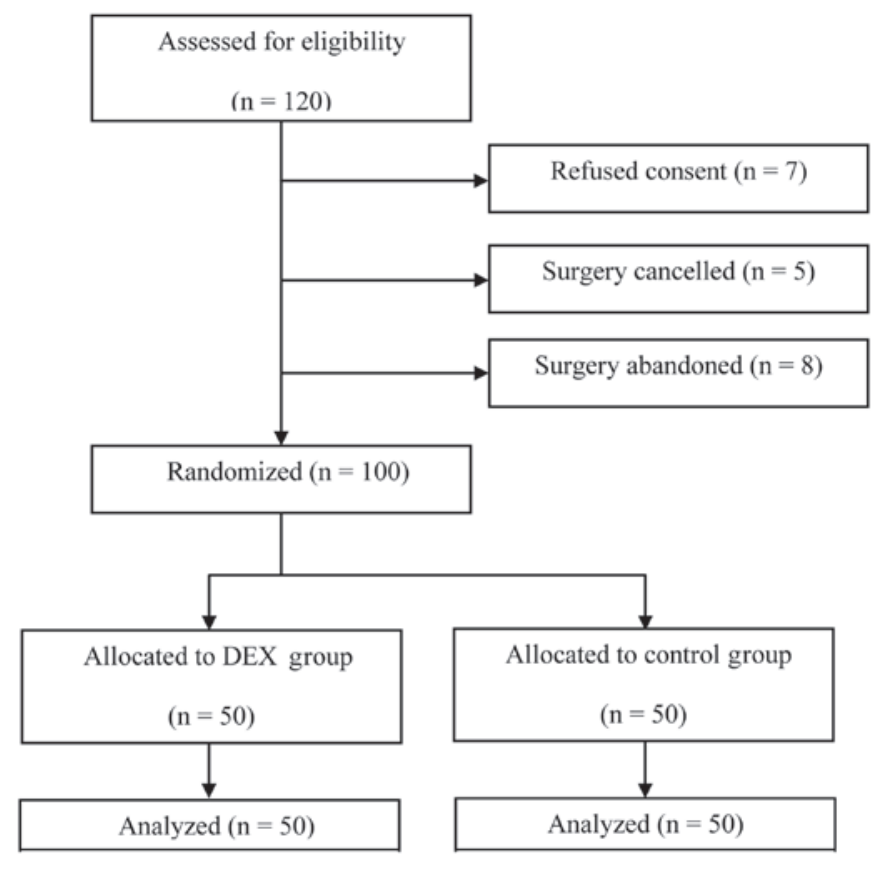

Figure 1. Flow chart of study inclusion. DEX, dexmedetomidine.

elevated (Fig. 3C). At 2 days after surgery the three mediators returned to baseline levels for patients in the DEX group.

Comparison between patients with and without POCD. The patients were divided into two groups according to whether or not they developed POCD. The characteristics of the patients with POCD and without POCD are presented in Table IV. Those who developed POCD had a longer disease duration (mean, 3.5 vs. 2.5 years, $\mathrm{P}=0.009$ ) and more co-morbid diseases $(\mathrm{P}=0.017)$. Other characteristics, including MMSE scores, age, weight, height and ASA score were similar in patients with or without POCD. Plasma levels of IL-1 $\beta$, IL-6, and CRP on day 1 after surgery were significantly higher in patients with POCD $(\mathrm{P}<0.05)$, while surgical duration, anesthesia duration and the amount of fluid infusion were similar between the patients with and without POCD. Hemodynamic data were also comparable in the patients with and without POCD (data not shown).

\section{Discussion}

Previous studies have shown that patients $>60$ years of age may have a higher risk of developing POCD. Moller et al (6) reported that POCD was observed in $25.8 \%$ of elderly patients 1 week after surgery and $9.9 \%$ of patients 3 months after surgery (22). Anesthesia may also be one of the causes of POCD. Unlike general anesthesia, local anesthesia does not have the potential advantage of reducing the incidence of POCD (23). General anesthetics, particularly inhaled agents are likely to be associated with POCD (24-27). The present study revealed that $31 \%$ of the elderly patients undergoing selective LC developed POCD $24 \mathrm{~h}$ after surgery. The study also demonstrated that the incidence of POCD was markedly reduced by intravenously administered dexmedetomidine (20 vs. $42 \%$ in the control group; $\mathrm{P}<0.01$ ), which is consistent with a previous study (28). 
Table I. Baseline characteristics of patients and procedures in the DEX and control groups.

\begin{tabular}{|c|c|c|c|}
\hline Characteristics & DEX group & Control group & P-value \\
\hline Age, years & $69 \pm 5$ & $70 \pm 6$ & 0.556 \\
\hline Gender n/N (\% female) & $22 / 50(44)$ & $24 / 50(48)$ & 0.841 \\
\hline Body weight, $\mathrm{kg}$ & $59 \pm 7$ & $60 \pm 8$ & 0.519 \\
\hline Height, cm & $163 \pm 8$ & $164 \pm 7$ & 0.294 \\
\hline $\mathrm{BMI}, \mathrm{kg} / \mathrm{m}^{2}$ & $22 \pm 3$ & $22 \pm 3$ & 0.906 \\
\hline Disease duration, years & $3(2-3.5)$ & $3(2-3.6)$ & 0.561 \\
\hline No. of co-morbid diseases & $1(1-2)$ & $1(1-2)$ & 0.123 \\
\hline No. of medication at home & $2(1-3)$ & $2(1-3)$ & 0.587 \\
\hline $\mathrm{ASA}^{\mathrm{a}}$ group, $\mathrm{n} / \mathrm{N}(\%)$ & & & 0.912 \\
\hline Group I & $21 / 50(42)$ & $22 / 50(44)$ & \\
\hline Group II & $22 / 50(44)$ & $20 / 50(40)$ & \\
\hline Group III & $7 / 50(14)$ & $8 / 50(16)$ & \\
\hline Surgical duration, min & $59 \pm 8$ & $60 \pm 10$ & 0.734 \\
\hline Anesthetic duration, min & $77 \pm 9$ & $78 \pm 11$ & 0.524 \\
\hline \multicolumn{4}{|l|}{ Amount of fluid administered, ml } \\
\hline Ringer's lactate solution & $92 \pm 15$ & $90 \pm 15$ & 0.176 \\
\hline $6 \%$ hydroxyethyl starch & $576 \pm 45$ & $568 \pm 63$ & 0.314 \\
\hline Post-operative diagnosis, n/N (\%) & & & 0.953 \\
\hline Chronic cholecystitis & $23 / 50(46)$ & $22 / 50(44)$ & \\
\hline Gall bladder stone & 19/50 (38) & $20 / 50(40)$ & \\
\hline Gall bladder polyps & $5 / 50(10)$ & $6 / 50(12)$ & \\
\hline Biliary colic & $3 / 50(6)$ & $2 / 50(4)$ & \\
\hline
\end{tabular}

Data are presented as mean \pm standard deviation or mean (interquartile range); $\mathrm{n} / \mathrm{N}$ is number with the characteristic/total number. DEX, dexmedetomidine; BMI, body mass index; ${ }^{a}$ ASA, American Society of Anesthesiologist physical status classification system, range 1 (normal health) to 5 (moribund).
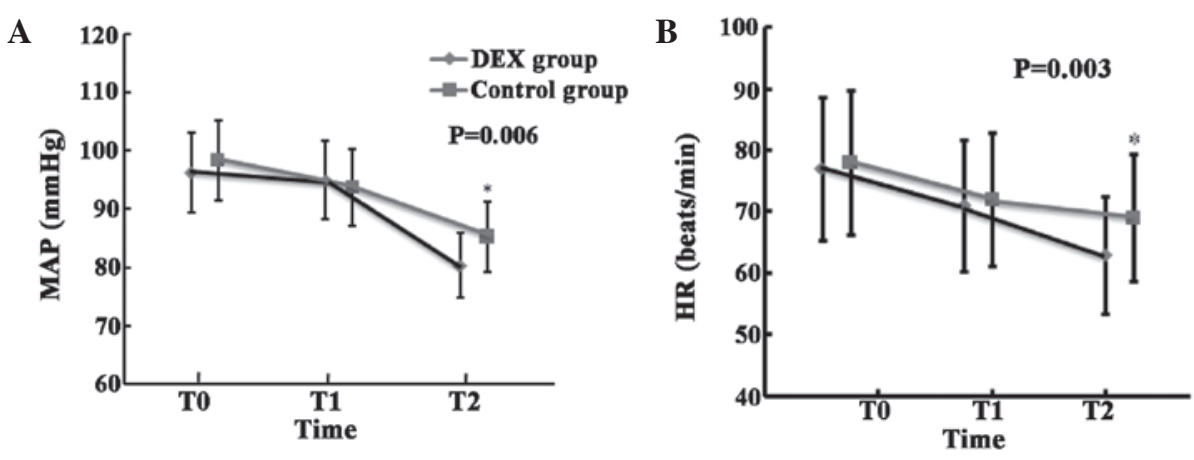

Figure 2. Differences in (A) MAP and (B) HR between the groups. "P<0.05 vs. control. T0, prior to induction of anesthesia; T1, 10 min after infusion of study drug; T2, at the end of peritoneal closure; HR, heart rate; MAP, mean arterial pressure; DEX, dexmedetomidine.

Although this current and previous research (28) revealed that DEX may provide neuroprotection in elderly patients following surgery, the mechanisms associated with this neuroprotective effect are not clear. It is well-known that anesthesia and surgical trauma, combined with primary disease requiring surgery and commodities constitute sources of stress to patients, which is also considered to be responsible for POCD $(6,8)$. The interaction of the neuroendocrine and sympathetic nervous systems promotes the release of inflammatory factors, particularly the nonspecific inflammatory reaction of the nervous system, likely promoting the development of POCD in elderly surgical patients (9-15). It has been demonstrated that the pathological processes of central nervous system disease are associated with inflammatory and immunological responses $(29,30)$. In addition to surgery, anesthetic techniques and the anesthetics themselves could trigger the release of stress hormones and cytokines. Factors such as IL-1 $\beta$, IL-6 and tumor necrosis factor- $\alpha$ (TNF- $\alpha)$, a cytokine with a wide bioactivity, can cross 
Table II. MMSE scores in the DEX and control groups.

\begin{tabular}{lccr}
\hline Time of test & DEX group & Control group & P-value \\
\hline T0 & $28.4 \pm 1.3$ & $28.3 \pm 1.4$ & 0.710 \\
T1 & $27.3 \pm 2.3$ & $24.3 \pm 2.3$ & $<0.001$ \\
T2 & $27.9 \pm 1.7$ & $26.7 \pm 1.9$ & 0.012 \\
T3 & $28.0 \pm 1.6$ & $28.2 \pm 1.3$ & 0.604 \\
\hline
\end{tabular}

Data are reported as mean \pm standard deviation. DEX, dexmedetomidine; MMSE, Mini Mental-State Examination; T0, prior to induction of anesthesia (baseline); T1, $6 \mathrm{~h}$ after surgery; T2, 1 day after surgery; T3, 2 days after surgery.

Table III. Degree of cognitive impairment $24 \mathrm{~h}$ after surgery.

\begin{tabular}{lccc}
\hline & \multicolumn{2}{c}{ Rate of impairment, $\mathrm{n} / \mathrm{N}(\%)$} & \\
\cline { 2 - 3 } $\begin{array}{l}\text { Degree of } \\
\text { impairment }\end{array}$ & DEX group & Control group & P-value \\
\hline Mild & $9 / 50(18)$ & $19 / 50(38)$ & 0.026 \\
Moderate & $1 / 50(2)$ & $2 / 50(4)$ & 0.558 \\
Severe & 0 & 0 & - \\
\hline
\end{tabular}

According to the Mini Mental-State Examination score: 21-27, mild cognitive impairment; 9-21, moderate cognitive impairment; and $<9$, severe cognitive impairment. $\mathrm{n} / \mathrm{N}$ is number with the impairment/total number. DEX, dexmedetomidine.

the blood brain barrier, promote brain cell permeability, and cause an inflammatory reaction in the central nervous system, thereby affecting the functioning of synaptic connections, resulting in cognitive function damage. Prolonged elevation of levels of IL-1 $\beta$ in the brain, specifically in the hippocampus, may be responsible for learning and memory impairments as such an elevation has been shown to damage hippocampal long-term potentiation (31). TNF- $\alpha$ and IL-1 $\beta$ can stimulate actin in the brain and cause actin regeneration, which plays a key role in the process of nerve degeneration (32). Clinical studies have shown that the levels of CRP, TNF- $\alpha$, IL- 6 and IL-1 increase markedly postoperatively in elderly hip-fracture patients $(33,34)$. The levels of cytokines, particularly IL-6, have been observed to increase significantly in patients with impaired mental status $(33,34)$. Compared with the major traumatic surgeries such as open thoracotomy and laparotomy or orthopedic surgery, LC is considered to be a minor surgery. Recently Kang et al (35) reported that LC also induced the release of inflammatory mediators. Thus, for elderly patients, the inhibition of the release of inflammatory factors associated with the LC procedure is important for the reduction of postoperative complications, including POCD.

These results indicate that dexmedetomidine can decrease the incidence of POCD in elderly patients; however, the following caveats should be considered. The present study found that dexmedetomidine administration decreased the incidence of mild cognitive impairment $24 \mathrm{~h}$ after surgery, which was consistent with the findings in Chen et al (28);
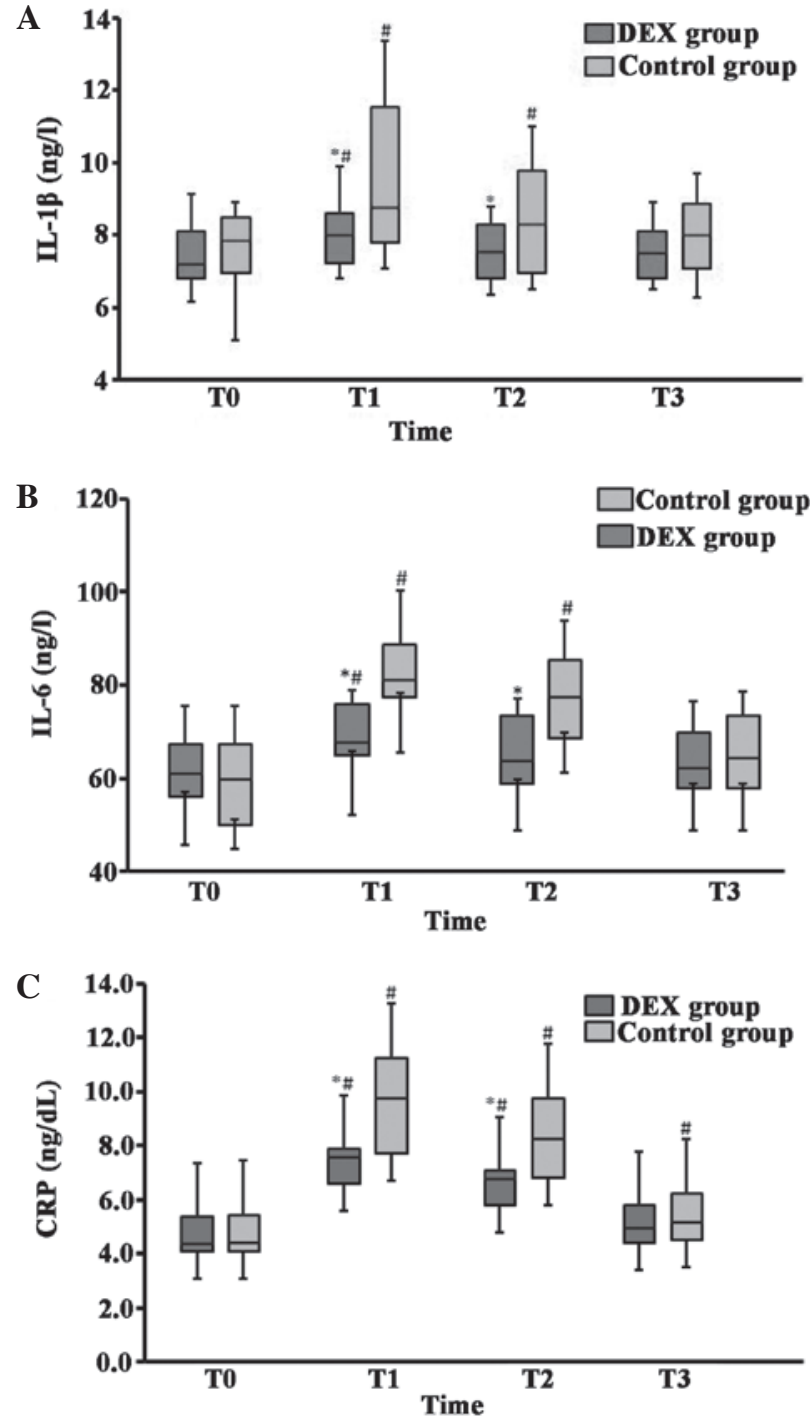

Figure 3. Comparison of cytokine (A) IL-1 $\beta$, (B) IL-6 and (C) CRP concentrations between the DEX and control groups. The box-plots display interquartile range (box), the median (line in the middle of the box) and 10th and 90th percentiles (error bars). ${ }^{*} \mathrm{P}<0.05$ vs. the control group. ${ }^{\#} \mathrm{P}<0.05$ vs. baseline (T0). T0, prior to induction of anesthesia; T1, $6 \mathrm{~h}$ after surgery; T2, 1 day after surgery; T3, 2 days after surgery. DEX, dexmedetomidine; IL, interleukin; CRP, C-reactive protein.

however, the difference from the controls did not reach statistical significance in the previous study (28). The differences in the characteristics, including older age and more severe pre-operative morbidity in the current study. The study by Kang et al (35) showed that the levels of pro-inflammatory cytokines remained almost the same during and $1 \mathrm{~h}$ after the surgery for patients with a mean age of $<45$ years, ASA I or II undergoing LC surgery in the control group. The present data revealed that the levels of IL-1 $\beta$, IL- 6 and CRP were higher $6 \mathrm{~h}$ postoperatively in the control group than in the DEX group. The possible contributing factors include the condition, age and other concomitant diseases of the patients. Further study is necessary to confirm these.

The aim of the present study was to investigate the effects of dexmedetomidine on the incidence of POCD through the suppression of harmful inflammatory mediators. It was found that the levels of IL-1 $\beta$, IL- 6 and CRP were attenuated by the 
Table IV. Comparison between the patients who developed POCD one day after surgery and the patients without POCD.

\begin{tabular}{|c|c|c|c|}
\hline Characteristic & No POCD & POCD & P-value \\
\hline $\mathrm{N}$ & 72 & 28 & - \\
\hline Gender $\mathrm{n} / \mathrm{N}$ (\% female) & $33 / 72(46)$ & $13 / 28(46)$ & 0.830 \\
\hline Age, years & $69 \pm 5$ & $69 \pm 6$ & 0.770 \\
\hline ASA group, n/N (\%) & & & 0.208 \\
\hline Group I & $33 / 72(46)$ & $10 / 28(36)$ & - \\
\hline Group II & $31 / 72(43)$ & 11/28 (39) & - \\
\hline Group III & $8 / 72(11)$ & $7 / 28(25)$ & - \\
\hline Weight, kg & $62 \pm 7$ & $61 \pm 8$ & 0.342 \\
\hline Height, cm & $164 \pm 7$ & $163 \pm 6$ & 0.667 \\
\hline $\mathrm{BMI}, \mathrm{kg} / \mathrm{m}^{2}$ & $23.3 \pm 3.1$ & $22.8 \pm 3.7$ & 0.538 \\
\hline Disease duration, years & $2.5(2-3.5)$ & $3.5(2.5-4)$ & 0.009 \\
\hline No. of co-morbid diseases & $1(1-2)$ & $2(1-2)$ & 0.017 \\
\hline No. of medication at home & $2(1-3)$ & $2(1-3)$ & 0.544 \\
\hline Surgical duration, min & $61 \pm 9$ & $60 \pm 10$ & 0.699 \\
\hline Anesthesia duration, min & $78 \pm 10$ & $78 \pm 9$ & 0.866 \\
\hline $6 \%$ hydroxyethyl starch, ml & $87 \pm 17$ & $91 \pm 12$ & 0.227 \\
\hline Ringer's lactate, $\mathrm{ml}$ & $555 \pm 65$ & $568 \pm 62$ & 0.364 \\
\hline IL-1 $\beta, \mathrm{ng} / 1$ & $7.8 \pm 1.6$ & $8.9 \pm 1.9$ & 0.013 \\
\hline IL-6, ng/l & $69.0 \pm 11.6$ & $77.7 \pm 16.5$ & 0.015 \\
\hline CRP, mg/dl & $7.1 \pm 1.1$ & $8.1 \pm 1.8$ & 0.010 \\
\hline Baseline MMSE & $28.4 \pm 1.6$ & $28.2 \pm 1.6$ & 0.626 \\
\hline
\end{tabular}

Data are presented as mean \pm standard deviation or mean (interquartile range). $\mathrm{n} / \mathrm{N}$ is number with charareristic/total number. DEX, dexmedetomidine; POCD, postoperative cognitive dysfunction; BMI, body mass index; IL, interleukin; CRP, C-reactive protein; MMSE, Mini Mental-State Examination; ASA, American Society of Anesthesiologist physical status classification system, range 1 (normal health) to 5 (moribund).

administration of dexmedetomidine to elderly patients undergoing LC, although at $6 \mathrm{~h}$ following surgery, the plasma levels of IL-1 $\beta$ and IL-6 also were significantly increased compared with baseline data in patients in the DEX group. The concentration of CRP was also higher than baseline 1 day following the procedure in the DEX group. When a comparison was made between the DEX and control groups, the baseline values were comparable, and the concentrations of IL-1 $\beta$, IL-6 and CRP were significantly lower in the DEX group at $6 \mathrm{~h}$ and 1 day following surgery. These findings indicated that dexmedetomidine administration during surgery can reduce the inflammatory reaction associated with surgery and anesthetics peri-operatively. When the patients were separated into two groups according to whether they developed POCD by day 1 postoperatively, it was found that the levels of IL-1 $\beta$, IL-6 and CRP were significantly higher in patients with POCD. Disease duration and the number of co-morbid diseases were contributing factors to POCD development. Additionally, it was found that MMSE scores were significantly lower in the control group $6 \mathrm{~h}$ and 1 day following surgery. Such changes in inflammatory factors were consistent with the lower MMSE scores, suggesting that DEX reduced the incidence of POCD through the suppression of the inflammatory response. To the best of our knowledge, the present study is the first to investigate the association between the development of POCD and pro-inflammatory cytokines. These findings are consistent with the hypothesis that dexmedetomidine can decrease systemic inflammatory factors and, therefore, reduce the incidence of POCD.

The results of the current study with elderly patients undergoing minor surgery are consistent with those of previous studies, indicating that dexmedetomidine decreases cytokine secretion $(36,37)$. The detailed mechanism of the anti-inflammatory reaction of dexmedetomidine during surgery remains unclear. The suppression of the production of cytokines may be associated with pre-synaptic $\alpha 2$-adrenoceptor-related mechanisms. The binding of dexmedetomidine to $\alpha 2$-adrenoceptors present in presynaptic membranes is expected to cause lipopolysaccharide-induced apoptosis of lymphocytes and monocytes/macrophages, thus reducing the amount of inflammatory cytokines $(36,37)$. Additionally, subsequent to dexmedetomidine combining with the post-synaptic $\alpha 2$-adrenoceptors, the majority of which are thought to be post-synaptic in the spinal cord, the concentration of norepinephrine increases, leading to negative immunoregulatory effects through suppression 
of the production of proinflammatory cytokines such as IL-6 and TNF- $\alpha(36,38)$. Surgery-associated tissue damage stimulates the inflammatory cascade, including nociceptive and inflammatory responses, accompanied by elevation of cytokines (39). Dexmedetomidine decreases the projection of ascending nociceptive neurons in the dorsal horn of the spinal cord, and then produces an analgesic effect. It is likely that dexmedetomidine reduces cytokine levels by inhibiting nociception. $\alpha 2$-adrenoceptor agonists are also considered to stimulate the neural microenvironment and change the phenotype of proinflammatory cytokines, reducing the expression of inflammatory cytokines (40). In the present study, dexmedetomidine administration decreased the HR and MAP at the end of the surgical procedure, and this may have been induced by a central sympatholytic effect mediated by $\alpha 2$-adrenoceptor activation and the consequent activation of cholinergic activity, reducing the production of cytokines through the cholinergic anti-inflammatory pathway (38). Therefore, it appears that dexmedetomidine may exert its anti-inflammatory effect through a variety of mechanisms.

Limitations of the present study included the fact that the plasma concentrations of anti-inflammatory cytokines were not detected. Westhoff et al (12) stressed the role of decreased levels of anti-inflammatory cytokines in the occurrence of delirium, an acute impairment in attention and cognition. A future study will explore the effect of dexmedetomidine treatment on the balance of pro-inflammatory and anti-inflammatory cytokines.

In conclusion, treatment with dexmedetomidine during LC inhibits the secretion of pro-inflammatory cytokines, which may be beneficial for postoperative cognitive function in elderly patients. The present findings support the hypothesis that dexdetomidine reduces the incidence of POCD by downregulating the inflammatory response. Dexmedetomidine can be safely applied during anesthesia in a straightforward surgery such as LC in elderly patients. It may be considered as a method to prevent POCD in elderly patients.

\section{Acknowledgements}

The authors would like to thank Dr. Xue-Jun Dong, the physician of Shaoxing People's Hospital, for assistance in the measurement of cytokines. This project was supported by Zhejiang Medical Association, (grant no. 2012ZYC-A72) and Wenling Science and Technology bureau (grant no. w112114).

\section{References}

1. Tang JX: The characteristics and operation for elderly patients with inguinal hernia. Lin Chuang Er Bi Yan Hou Tou Jing Wai Ke Za Zhi 16: 224-226, 2008 (In Chinese).

2. Monk TG, Weldon BC, Garvan CW, Dede DE, van der Aa MT, Heilman KM and Gravenstein JS: Predictors of cognitive dysfunction after major noncardiac surgery. Anesthesiology 108: $18-30,2008$

3. Steinmetz J, Christensen KB, Lund T, Lohse N and Rasmussen LS; ISPOCD Group: Long-term consequences of postoperative cognitive dysfunction. Anesthesiology 110: 548-555, 2009.

4. Cibelli M, Fidalgo AR, Terrando N, Ma D, Monaco C, Feldmann M, Takata M, Lever IJ, Nanchahal J, Fanselow MS and Maze M: Role of interleukin-1beta in postoperative cognitive dysfunction. Ann Neurol 68: 360-368, 2010.

5. Sauër AM, Kalkman C and van Dijk D: Postoperative cognitive decline. J Anesth 23: 256-259, 2009.
6. Moller JT, Cluitmans P, Rasmussen LS, Houx P, Rasmussen H, Canet J, Rabbitt P, Jolles J, Larsen K, Hanning CD et al: Long-term postoperative cognitive dysfunction in the elderly ISPOCD1 study. ISPOCD investigators. International Study of Post-Operative Cognitive Dysfunction. Lancet 351: 857-861, 1998.

7. McDonagh DL, Mathew JP, White WD, Philips-Bute B, Laskowitz DP, Podgoreanu MV and Newman MF; Neurologic Outcome Research Group: Cognitive function after major noncardiac surgery, apolipoprotein E4 genotype, and biomarkers of brain injury. Anesthesiology 112: 852-859, 2010.

8. Rasmussen LS: Postoperative cognitive dysfunction: Incidence and prevention. Best Pract Res Clin Anaesthesiol 20: 315-330, 2006.

9. Wan Y, Xu J, Ma D, Zeng Y, Cibelli M and Maze M: Postoperative impairment of cognitive function in rats: A possible role for cytokine-mediated inflammation in the hippocampus. Anesthesiology 106: 436-443, 2007.

10. Li YC, Xi CH, An YF, Dong WH and Zhou M: Perioperative inflammatory response and protein S-100 $\beta$ concentrations - relationship with post-operative cognitive dysfunction in elderly patients. Acta Anaesthesiol Scand 56: 595-600, 2012.

11. Ji MH, Yuan HM,Zhang GF, Li XM, Dong L, Li WY,Zhou ZQ and Yang JJ: Changes in plasma and cerebrospinal fluid biomarkers in aged patients with early postoperative cognitive dysfunction following total hip-replacement surgery. J Anesth 27: 236-242, 2013.

12. Westhoff D, Witlox J, Koenderman L, Kalisvaart KJ, de Jonghe JF, van Stijn MF, Houdijk AP, Hoogland IC, Maclullich AM, van Westerloo DJ, et al: Preoperative cerebrospinal fluid cytokine levels and the risk of postoperative delirium in elderly hip fracture patients. J Neuroinflammation 10: $122,2013$.

13. Xie G, Zhang W, Chang Y and Chu Q: Relationship between perioperative inflammatory response and postoperative cognitive dysfunction in the elderly. Med Hypotheses 73: 402-403, 2009.

14. Fidalgo AR, Cibelli M, White JP, Nagy I, Maze M and Ma D: Systemic inflammation enhances surgery-induced cognitive dysfunction in mice. Neurosci Lett 498: 63-66, 2011

15. Peng L, Xu L and Ouyang W: Role of peripheral inflammatory markers in postoperative cognitive dysfunction (POCD): A meta-analysis. PLoS One 8: e79624, 2013.

16. Mantz J, Josserand J and Hamada S: Dexmedetomidine: New insights. Eur J Anaesthesiol 28: 3-6, 2011.

17. Su F and Hammer GB: Dexmedetomidine: Pediatric pharmacology, clinical uses and safety. Expert Opin Drug Saf 10: 55-66, 2011.

18. Shukry M and Miller JA: Update on dexmedetomidine: Use in nonintubated patients requiring sedation for surgical procedures. Ther Clin Risk Manag 6: 111-121, 2010.

19. Monk TG and Price CC: Postoperative cognitive disorders. Curr Opin Crit Care 17: 376-381, 2011.

20. Folstein MF, Folstein SE and McHugh PR: "Mini-mental state". A practical method for grading the cognitive state of patients for the clinician. J Psychiatr Res 12: 189-198, 1975.

21. Newman SP: Analysis and interpretation of neuropsychologic tests in cardiac surgery. Ann Thorac Surg 59: 1351-1355, 1995.

22. Bedford PD: Adverse cerebral effects of anaesthesia on old people. Lancet 269: 259-263, 1955.

23. Rasmussen LS: Postoperative cognitive dysfunction: Incidence and prevention. Best Pract Res Clin Anaesthesiol 20: 315-330, 2006.

24. Zurek AA, Bridgwater EM and Orser BA: Inhibition of $\alpha 5$ $\gamma$-aminobutyric acid type A receptors restores recognition memory after general anesthesia. Anesth Analg 114: 845-855, 2012.

25. Callaway JK, Jones NC and Royse CF: Isoflurane induces cognitive deficits in the Morris water maze task in rats. Eur J Anaesthesiol 29: 239-245, 2012.

26. Le Freche H, Brouillette J, Fernandez-Gomez FJ, Patin P, Caillierez R, Zommer N, Sergeant N, Buée-Scherrer V, Lebuffe G, Blum D and Buée L: Tau phosphorylation and sevoflurane anesthesia: An association to postoperative cognitive impairment. Anesthesiology 116: 779-787, 2012.

27. Kavanagh T and Buggy DJ: Can anaesthetic technique effect postoperative outcome? Curr Opin Anaesthesiol 25: 185-198, 2012.

28. Chen J, Yan J and Han X: Dexmedetomidine may benefit cognitive function after laparoscopic cholecystectomy in elderly patients. Exp Ther Med 5: 489-494, 2013. 
29. Emsley HC and Hopkins SJ: Acute ischaemic stroke and infection: Recent and emerging concepts. Lancet Neurol 7: 341-353, 2008.

30. Emsley HC, Smith CJ, Gavin CM, Georgiou RF, Vail A, Barberan EM, Hallenbeck JM, del Zoppo GJ, Rothwell NJ, Tyrrell PJ and Hopkins SJ: An early and sustained peripheral inflammatory response in acute ischaemic stroke: Relationships with infection and atherosclerosis. J Neuroimmunol 139: 93-101, 2003.

31. Barrientos RM, Frank MG, Hein AM, Higgins EA, Watkins LR, Rudy JW and Maier SF: Time course of hippocampal IL-1 beta and memory consolidation impairments in aging rats following peripheral infection. Brain Behav Immun 23: 46-54, 2009.

32. Salminen A, Ojala J, Kauppinen A, Kaarniranta K and Suuronen T: Inflammation in Alzheimer's disease: Amyloid-beta oligomers trigger innate immunity defence via pattern recognition receptors. Prog Neurobiol 87: 181-194, 2009.

33. Barrientos RM, Higgins EA, Biedenkapp JC, Sprunger DB Wright-Hardesty KJ, Watkins LR, Rudy JW and Maier SF: Peripheral infection and aging interact to impair hippocampal memory consolidation. Neurobiol Aging 27: 723-732, 2006.

34. Beloosesky Y, Hendel D, Weiss A, Hershkovitz A, Grinblat J, Pirotsky A and Barak V: Cytokines and C-reactive protein production in hip-fracture-operated elderly patients. J Gerontol A Biol Sci Med Sci 62: 420-426, 2007.
35. Kang SH, Kim YS, Hong TH, Chae MS, Cho ML, Her YM and Lee J: Effects of dexmedetomidine on inflammatory responses in patients undergoing laparoscopic cholecystectomy. Acta Anaesthesiol Scand 57: 480-487, 2013.

36. Maes M, Lin A, Kenis G, Egyed B and Bosmans E: The effects of noradrenaline and alpha-2 adrenoceptor agents on the production of monocytic products. Psychiatry Res 96: 245-253, 2000.

37. Szelényi J, Kiss JP and Vizi ES: Differential involvement of sympathetic nervous system and immune system in the modulation of TNF-alpha production by alpha-2- and beta-adrenoceptors in mice. J Neuroimmunol 103: 34-40, 2000.

38. Klimscha W, Tong C and Eisenach JC: Intrathecal alpha 2-adrenergic agonists stimulate acetylcholine and norepinephrine release from the spinal cord dorsal horn in sheep. An in vivo microdialysis study. Anesthesiology 87: 110-116, 1997.

39. Shavit Y, Fridel K and Beilin B: Postoperative pain management and proinflammatory cytokines: Animal and human studies. J Neuroimmune Pharmacol 1: 443-451, 2006.

40. Devor M, Jänig W and Michaelis M: Modulation of activity in dorsal root ganglion neurons by sympathetic activation in nerve-injured rats. J Neurophysiol 71: 38-47, 1994. 